\title{
Empirical Analysis of Intelligent Handover Mechanism using Numerous Fuzzy Strategies
}

\author{
Sandeep Sivvam, Neeraja S, Jyothi Lakshmi T S, Dharma Raj C
}

\begin{abstract}
In the communication world, each and every individual belongs different types of communication modes to establish their data transmission needs around globe. Most preferable and well known communication medium is a Wireless Communication Mode, which provides stability and standardization to users to make their communication perfect. However, the communication lacking occurs due to low-latency and energy oriented issues, which indirectly affects the overall network throughput and it leads the overall network lifetime to low. So, there is a necessity to introduce a new algorithm to produce a stabilized platform to satisfy the user's communication needs with proper Quality-of-Service (QoS) norms. A new system is introduced, which is helpful to achieve high throughput and energy efficient communication between clusters by using fuzzy laws. In this system, Genetic Algorithm is used to generate Optimized Solutions with high Accuracy as well as it is used to reduce number of handovers by means of identify the energy level of each member nodes and design the pathway for communication according to that. And if dynamically generates the Node Analyzer (NA) for identifying the best nodes for communication and using that way the communication is established. The main concept in the proposed approach is achieved by means of designing a new algorithm called "Fuzzy Logic based Handover Decision Algorithm (FLHDA)", which provides better efficiency by means of integrating the following three different approaches such asFuzzification Stage, Fuzzy Inference Stage andDeffuzification Stage. For all the entire proposed approach guarantees that the outcome assures the Quality-of-Service, which is achieved by means of enhanced throughput, minimized end to end delay and energy improvements with less consumption strategies.
\end{abstract}

Keywords: Wireless Communication, Handover, Fuzzy Strategies, Node Analyzer, Fuzzy Logic based Handover Decision Algorithm, FLHDA, Fuzzification Stage, Fuzzy Inference Stage andDeffuzification Stage, Quality-of-Service (QoS).

\section{INTRODUCTION}

$\mathrm{T}$ he participation of Wireless Network Communications over data transmission environments are growing

Revised Manuscript Received on December 13, 2019.

* Correspondence Author

Sandeep Sivvam*, Assistant Professor, Department of ECE Engineering \& Technology Program, Gayatri Vidya Parishad College for Degree and PG Courses (A), Rushikonda, Visakhapatnam, India.Email: sandeepsivvam@gmail.com

Neeraja S, Assistant Professor, Department of ECE, GITAM Institute of Technology, Visakhapatnam, India. Email: gitamneeraja@gmail.com

Jyothi Lakshmi T S, Associate Professor, Department of ECE, Engineering \& Technology Program, Gayatri Vidya Parishad College for Degree and PG Courses (A), Rushikonda, Visakhapatnam, India.Email: jyoths.lakshmi@gmail.com

Dharma Raj C, Professor \& Principal (I/c), Department of ECE, GITAM Institute of Technology, Visakhapatnam, India. Email: dharmaraj.cheruku@gitam.edu day-by-day and provides lots of features to the customers to achieve their communication needs properly without any interruption.

There are various contrasting situations such as battlefield communications, emergency operations and rescue where wireless communication platform can be enabled by Self-organizing network configuration. In an uncertain manner the change of topology is caused by mobility and self-organizing features of wireless communication networks. Normally for data transmission each wireless node which has limited transmission range will have to take assistance from neighboring nodes.Therefore, the performance of wireless communication networks mostly depends on the consistent routing amongst nodes. While practical implementations there are lots of network routing protocols available and all can provide assurance over secure and reliable routing over communication, but, routing protocols are introduced with a probabilistic prediction that all the nodes are completelyreliable and are ready to cooperate with each other.

Thus, they are susceptible to routing disruption attackers that are not cooperative or disobey the routing rules. The network routing pathway selection over Wireless Communication Network model is a critical task in network scenario over communication, because of the mobility and absence of fixed region. This kind of routing disruption intruders can stealthily choose any aforesaid attack pattern that causelarge packet loss. Due to the open nature of wireless communication networks, it is relatively common that some malevolent nodes may hide in the network and hence drops the packets so as to save the energy or disrupt the network functionality. These are all the major factors needs to concentrate more in the proposed system and plan to design the robust protocol and strategy to avoid this kind of issues over mobile network environment. In this system, an advanced routing protocol is used to establish the communication between source/sender and destination/receiver efficiently, which is called a Dynamic Source Routing (DSR) protocol, in which this routing protocol is used to assure full defense and fault-free mechanisms over wireless network communication scenario. A most important feature of the DSR is that it is not getting compromised at any level of internal and external intrusions present into the system, which always trying to eliminate that in scratch and done that successfully in outcome. 


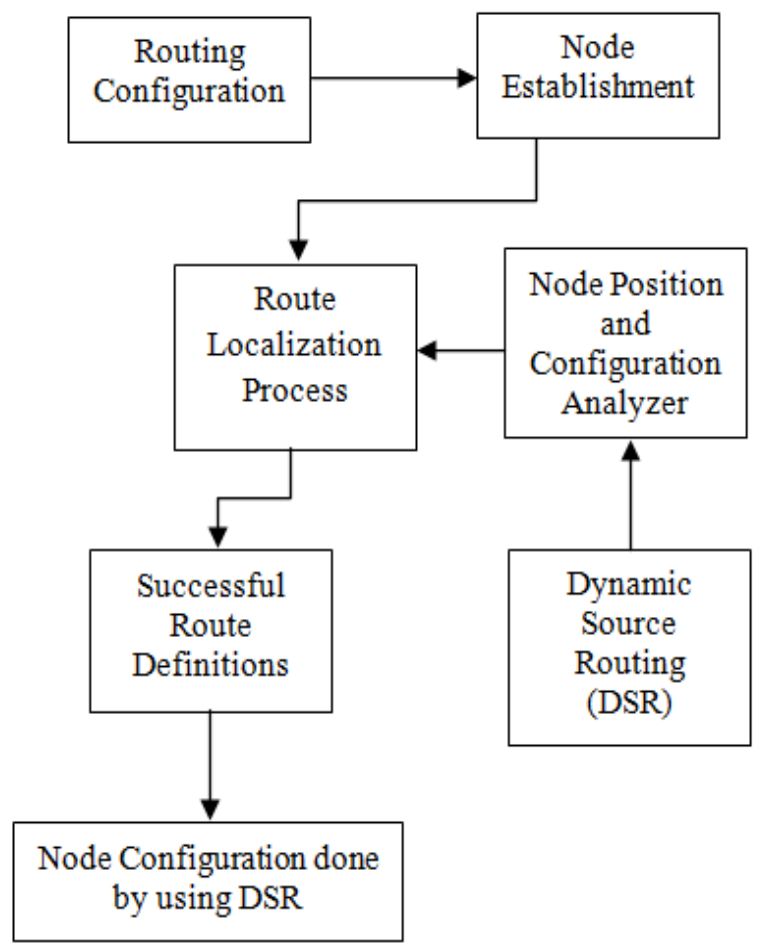

Fig.1 DSR Node Configuration Overview

Yet in wireless communication platform in order to transmit the data/information requires better efficiency to throughout needs and energy perception which is achieved by using fuzzy philosophies. The information over wireless communication and its address needs to be maintained into the access point and transferring that data is happened from source node to destination, so that even the service provider cannot retrieve the data and the retrieval time and it is used for further processing. So that the resulting approaches of Quality-of-Service (QoS) is solved using the following three different energetic stages of principles, such as: (i) Fuzzification Stage, (ii) Fuzzy Inference Stage and (iii) Deffuzification Stage. By considering these approaches, newprocedure/algorithm is formed so as to overcome the concerns such as throughput lacking, energy efficiency, responsive ability lacking and poor-battery lifetime called Fuzzy Logic based Handover Decision Algorithm (FLHDA). This proposed procedure/algorithm strenuously cares about computational overhead in wireless communication and solves the battery-lifetime and energy issues problems as well as provides faster responsiveness to wireless nodes with proper efficiency [2][6]. This methoderadicates the limitations of the poor network lifetime and provides QoS to the wireless communication platform and its users.

\section{SySTEMANALYSIS}

\section{A. Existing System Summary}

In the communication world wireless networks plays a vivacious role to satisfy the communication needs of clients with full of integrity and time efficient manner. In Wireless Communication mode, the communication pathway selection with proper throughput and energy efficiency are the two serious causes need to be analyzed and resolved. In regular wireless and wired network data transmission pathways are really drastic and easy to understand because of its sustainability, but in case of Wireless Network
Communication it is really hard to predict the location of the nodes and establish the communication pathway successfully at every time without any flaws. Due to the missing of stable environment, the Wireless Network Communication structure faces the routing issues and energy oriented issues always in communication scenario. This kind of routing issues always creates a complexity and challenges over network pathway establishments over Wireless Network Communication. There are various contrasting situations such as battlefield communications, emergency operations and rescue where wireless communication platform can be enabled by Self-organizing network configuration. In an uncertain manner the change of topology is caused by mobility and self-organizing features of wireless communication networks. Normally for data transmission each wireless node which has limited transmission range will have to take assistance from neighboring nodes.Consequently, the performance of wireless network communications mostly depends on the consistent routing among nodes. These different flaws in existing approach need to be resolved using some latest methodologies, so that a new procedure/algorithm is required to resolve this problems/issues in efficient manner.

\section{B. Summary of Proposed System}

The main intension of this proposed system is to prove the efficiency of Wireless Sensor Networks over wireless network communication architecture as well as the communication strength needs to be proved over wireless communication strategies. The traditional schemes to enhance the communication probabilities over wireless network communication are increasing day-by-day due to the communication needs of people and users. The complexity need to tolerate or resolved in this schema is to be clearly analyzed and rectified with this work by means of evaluate the classical communication methodologies. The existing network strategies follow communication pathway by means of shortest path identification and complete the communication based on node strength and success ratio, however the processing node getting down means the entire network spectrum needs to be changed and the new pathway is to be selected for further communication process. 


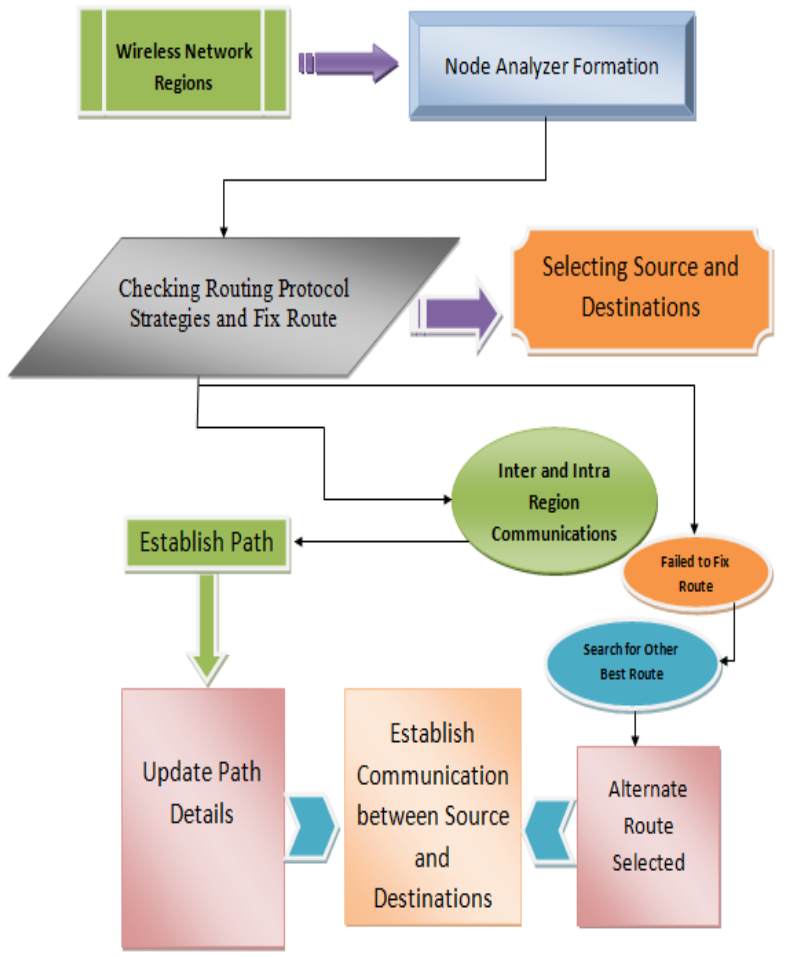

Fig.2 Architectural Perception of Proposed System

This processing delay causes severe damage over communication era over Wireless and Mobile Networks, because of the packet loss and network delay the entire network performance goes down in several stages as well as it replicates over the network lifetime. Because the node affection over the communication causes much time loss and it indirectly affects the other node strength as well. In this proposed methodology, a new strategy is applied called Fuzzy Logic based Handover Decision Algorithm (FLHDA), which concern on all the mentioned problems in past and provides the better communication strategies over results. The simulation is processed by means of the following way, such as the node formation is done dynamically by the user at runtime and the source/Sender and destination/Receiver node election process is also dynamically selected by the user at runtime. The proposed methodology FLHDA analyze the network pathway by means of intelligent strategy such as Route Request (RREQ) and Route Response (RREP), in which it analyze the next node strength by means of sending dummy packets to the next possible neighbor to analyze the Response strength of the neighbor, if the neighbor properly response for the request raised by the parental node, that will be elected as a next successful neighbor. If any case the neighbor node is not properly response for the request raised by the parental node, the nodes presented idly into the network will come into action and provides the sufficient energy or required bandwidth to the affected node and make the node recovered to perform successful communication without any failures. In order to avoid failures by this way means, the entire network lifetime won't be getting suffered at any case, because the idle nodes are remain safe as well as the affected node is also getting corrected by the relay process and in this situation there is no way for attacks in any terms. The proposed algorithm of FLHDA is associated with the protocol called Dynamic Source Routing (DSR) to provide better efficiency in results. The entire procedure gives guarantee for the better network lifetime, enhanced throughput, reduced network delay and improved performance over results.

\section{SYSTEM IMPLEMENTATION}

\section{A. Fuzzification Stage}

The fuzzification stage of this system is important to define the membership functions and also the clear estimation of distance ratios is attained via this fuzzification stage, as well as this implementation of fuzzification stage helps to identify the location of nodes easily. The fuzzification stage provides complete solution to identify the regions, node positions and distance between one another as well as helps to define the membership functions easily.

\section{Algorithm: Fuzzification Stage}

Input: Initialize the variables such as Node-ID, NS2 Path Area, Source and Destination.

Output: FL_HandOver.tr Trace File.

For $\{$ seti 0$\}\{\$ i<=39\}\{$ incri $\}\{$

set $\mathrm{k} 0$

set X1 [\$node_(\$i) set X_]

set y1 [\$node_( $\$$ i) set $\mathrm{Y}_{-}$]

for $\{$ set $\mathrm{j} 0\}\{\$ \mathrm{j}<=39\}\{$ incr $\mathrm{j}\}\{$

set x2 [\$node_(\$j) set $\left.X_{-}\right]$

set y2 [\$node_(\$j) set $\left.Y_{-}\right]$

set dis [expr sqrt(pow([expr \$x2 - \$x1],2)+pow([expr \$y2 \$y1],2))]

if $\{\$$ dis $<240 \& \& \$ \mathrm{i} !=\$ \mathrm{j}\}\{$ incr $\mathrm{k}$; set wt $\$ \mathrm{i}$

set $x 2[\operatorname{expr} \operatorname{int}(\$ x 1)] ;$ set y2 [expr int $(\$ y 1)]$

puts $\$ r$ "\$ilttt\$j|t|t\$x2lttt\$y2 $\backslash t \mid t \$ d i s "\}\}$

puts $\$ r$ "Neighbor_Nodes $\$ w t=\$ k "$ \}

\section{B. Fuzzy Inference Stage}

This Fuzzy Inference Stage of application helps to apply the Max-Min procedure to attain high possible and favorable results as an outcome. If-then rules are used to exploit the Max-min inference, leastphilological variables of the input parameters to presume the output of the system based on the set rules.It is the method of articulating the mapping from a given input to an output using fuzzy logic. The mapping affords a basis from which choices can be framed, or patterns determined Fuzzification of the input variables. Application of the fuzzy operator (AND or OR) in the antecedent. 


\section{Empirical Analysis of Intelligent Handover Mechanism using Numerous Fuzzy Strategies}

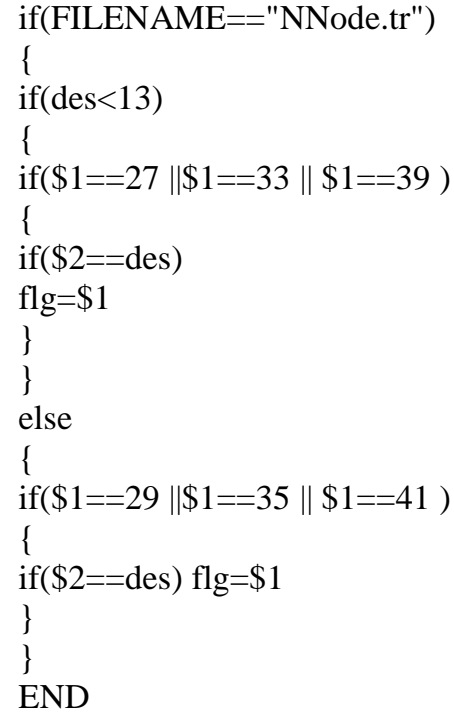

\section{Deffuzification Stage}

The Deffuzification Stage is used to obtain the traces of complete transactions between source and destinations. That particular trace file is named as Trace.tr in implementation and we attain all kind of transmission details between source and destination is trapped over there in implementation location.

\section{Algorithm: Deffuzification Stage}

set $\mathrm{f}$ [open Trace.tr $\mathrm{w}$ ]

\$ns_trace-all \$f

\$ns_use-newtrace

set namtrace [open Nam.nam w]

\$ns_namtrace-all-wireless \$namtrace \$val(x) \$val(y)

set topo [new Topography]

\$topo load_flatgrid \$val(x) \$val(y)

create-god \$val(nn)

\section{RESULTS AND DISCUSSION}

The simulation of the proposed system is implemented in Network Simulator 2 (NS2), that is used to design and implement the system with node creations and trace maintenance procedures with different algorithmic procedures.Fig. 3 shows that the simulation views of the proposed system.

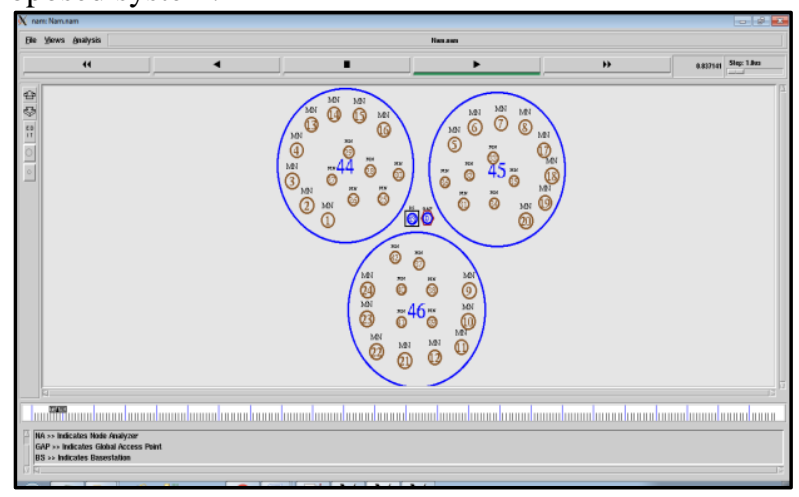

Fig.3 Node Formation

The Source and Destinations formation of the designed/proposed system implementation is shown inFig.4

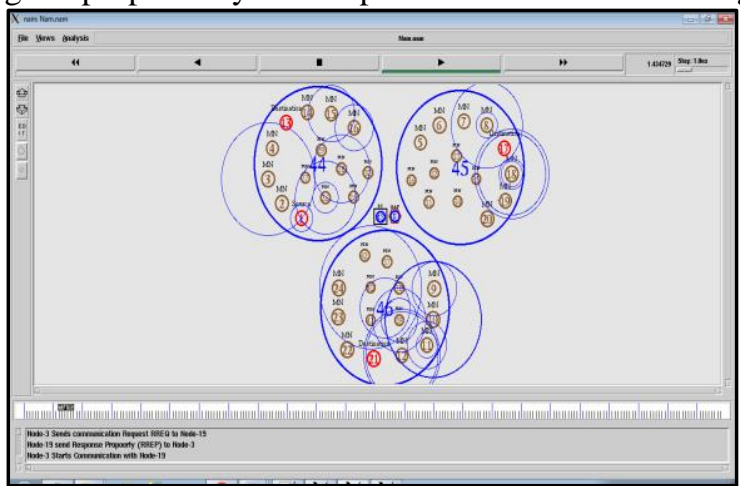

Fig.4 Source and Destination Formation

The Node Analyzer (NA) formation view of the proposed system is illustrated in Fig.5.

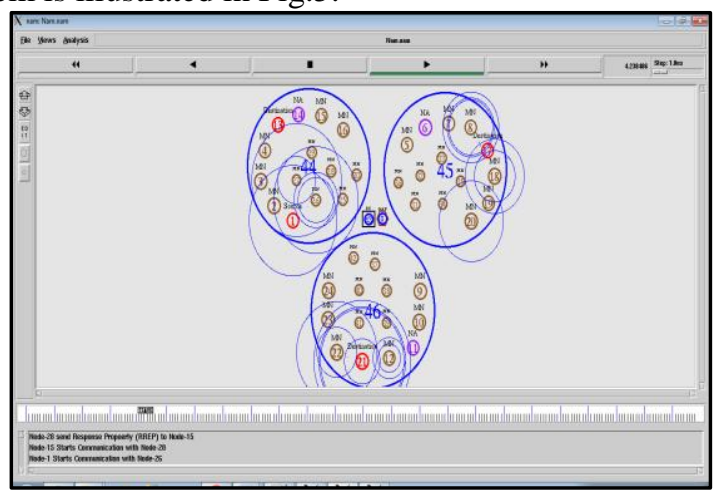

Fig.5 NA Formation

Fig.6 illustrates that the Communication Scenario and Node Movement Scenario of the proposed system.

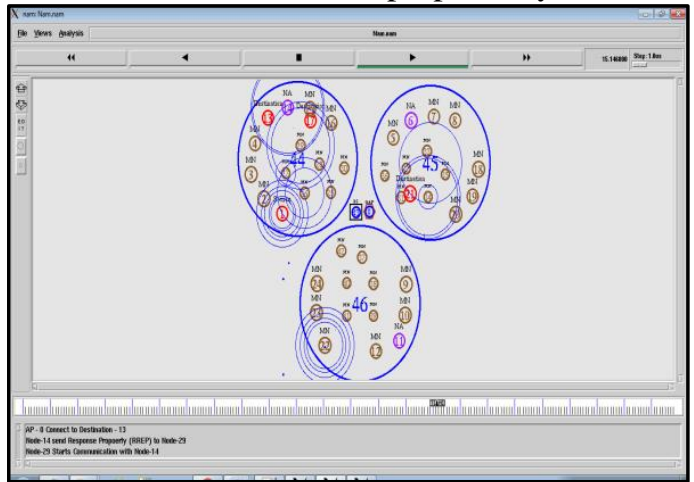

Fig.6 Node Movement Scenario and Communication Establishments

The abnormal node finding scenario of the proposed system is as shown in Fig.7

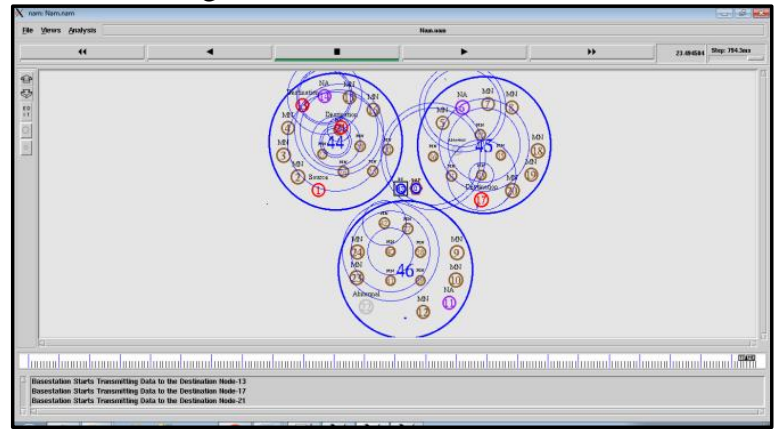

Fig.7 Abnormal Node Identification 
Fig.8 illustrates the Energy Efficiency graph view of the proposed system.

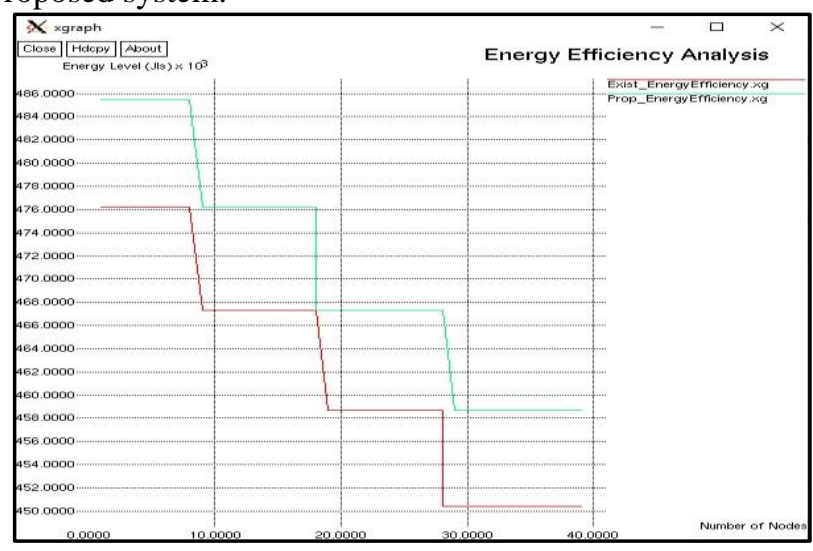

Fig.8 Energy Efficiency Analysis

Energy efficiency analysis is achieved through the following equation, which is explained below in clear [7][10][11].

$$
\mathrm{EE}=\operatorname{expr} \operatorname{int}(\$ s q 3 * \$ s q 4 * \$ s q 6) /(\$ s q 5-\$ s q 7+\$ s q 8)+1.5)
$$

Where EE is Energy Efficiency, sq3 is Transmission Packet Size, sq4 is Individual NodeStrength, sq6 is NodeMobilityRange, sq5 is Packet Transmission Speed, sq7 is AverageDelay and sq8 is ThresholdTimeStamp.

Fig.9 illustrates the Network Lifetime Analysis view of the proposed system.

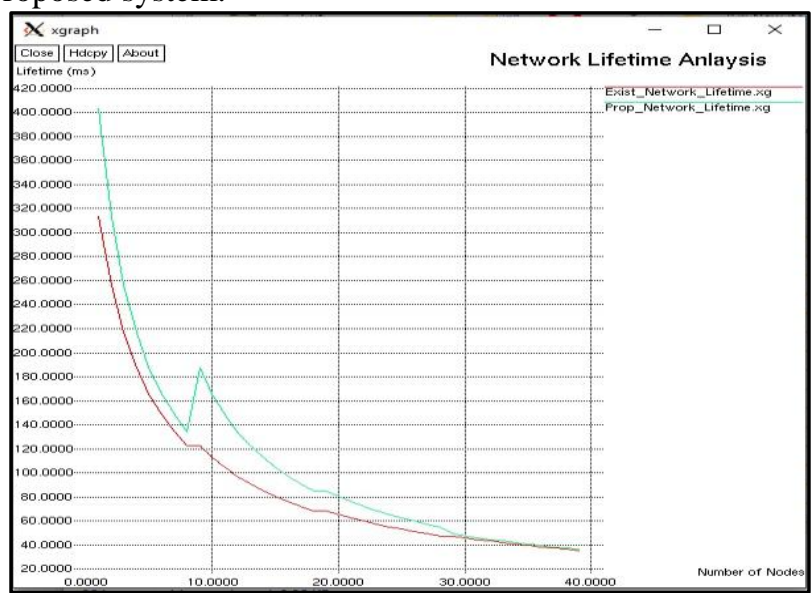

Fig.9 Network Lifetime Analysis

The network lifetime analysis is achieved by means of the following equation, which is explained below in clear [4][5][6][12].

\section{NLT $=\operatorname{expr}$ int $(\$ s q 3+\$ s q 4+\$ s q 5+\$ s q 6) /(\$ s q 7-2.5+\$ i)(2)$}

Where NLT is Network Lifetime, sq3 is Transmission Packet Size, sq4 is Individual NodeStrength, sq6 is NodeMobilityRange, sq5 is Packet Transmission Speed, sq7 is AverageDelay and ' $i$ ' is Node ID.

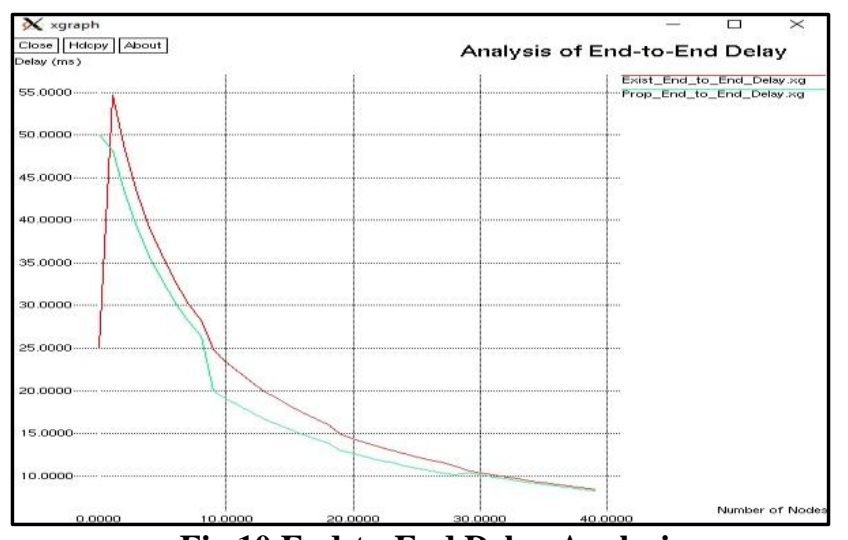

Fig.10 End-to-End Delay Analysis

End-to-End Network Delay Analysis view of the proposed system is illustrated in Fig.10. The End-to-End Network Delay analysis[1][2][3] is achieved by means of the following equation, which is explained below in clear.

\section{EED $=\operatorname{expr} \operatorname{int}(\$ s q 4+\$ s q 6+\$ s q 5) /(\$ s q 7+1.5+\$ i)(3)$}

Where EED is End-to-End Network Delay, sq4 is Individual NodeStrength, sq6 is NodeMobilityRange, sq5 is Packet Transmission Speed, sq7 is AverageDelay and ' $i$ ' is Node ID.

The Network Throughput level analysis of the proposed system is illustrated in Fig. 11

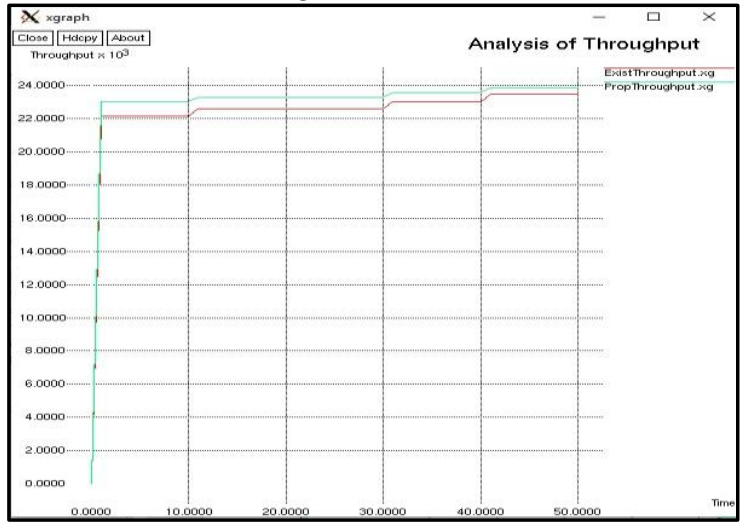

Fig.11 Network Throughput Analysis

The Network Throughput level analysis is measured by the following equation, which is explained below in clear [8][9][12].

$\mathrm{NT}=\operatorname{expr} \operatorname{int}(\$ s q 3 * \$ s q 4 * \$ s q 5) /(\$ s q 6+10.5)(4)$

Where NT is Network Throughput, sq3 is Transmission Packet Size, sq4 is Individual NodeStrength, sq6 is NodeMobilityRange, sq5 is Packet Transmission Speed.

\section{Conclusion}

In real-world scenario Wireless Communication Model has different range of applications and which it provides a huge change in communication nature. But the security issue has to be resolved in a careful manner before deploying the model.A new proposed scheme is introduced with DSR protocol called Fuzzy Logic based Handover Decision Algorithm (FLHDA), which is used to defend against various routing disruption attacks and ensures the trust enabled communication service with full of security schema is introduced here. 


\section{Empirical Analysis of Intelligent Handover Mechanism using Numerous Fuzzy Strategies}

By using this proposed approach, the following things are exposed with proper nature: (a) the trust analysis can be performed by taking advantage of mobility in self-detection mechanism, (b) fuzzification mechanism further improves the performance and boost the robustness of FLHDA and (c) the impact of wrong information given by the problematic nodes can be reduced by Deffuzication stage of FLHDA by enabling the nodes to explore various forms of networks. If the majority of nodes in the network are harmless, FLHDA can advance the Packet Delivery Ratio as well as improve the efficiency of communication protocol and eliminates the routing attacks.

\section{REFERENCES}

1. P.P. Bhattacharya, Application of artificial neural network in cellular handoff management, IEEE International Conference on Conference on Computational Intelligence and Multimedia Applications (ICCIMA), 2007, pp. 237-241.

2. N. Nasser, S. Guizani, E. Al-Masri, Middleware vertical handoff manager: a neural network-based solution, IEEE International Conference on Communications, ICC'07, 2007, pp. 5671-5676.

3. M. Okwori, E.N. Onwuka, A.M. Aibinu, O.C. Ugweje, Implementation of a locator-based route switching scheme for improved routing in proxy mobile IPv6, Int. J. Wirel. Microw. Technol. (2014) 119.

4. S.J. Wu, An intelligent handover decision mechanism for heterogeneous wireless networks, Sixth International Conference on Networked Computing and Advanced Information Management (NCM), 2010, pp. 688-693.

5. Q. Guo, J. Zhu, X. Xu, An adaptive multi-criteria vertical handoff decision algorithm for radio heterogeneous network, 2005 IEEE International Conference on Communications (ICC), 2005, pp. 2769-2773.

6. L. Barolli, F. Xhafa, A. Durresi, A. Koyama, A fuzzy-based handover system for avoiding ping-pong effect in wireless cellular networks, International Conference on Parallel Processing-Workshops ICPP-W'08, 2008, pp. 135-142.

7. R. Chai, J. Cheng, X. Pu, Q. Chen, Neural network based vertical handoff performance enhancement in heterogeneous wireless networks, 2011 7th International Conference on Wireless Communications, Networking and Mobile Computing (WiCOM), 2011, pp. 1-4.

8. P. Moungnoul, P. Pinprasert, T. Paungma, Handoff decision in cellular mobile system using neural network accessed online October 2016.

9. S. Kunarak, R. Sulessathira, E. Dutkiewicz, Vertical handoff with predictive RSS and dwell time, 2013 IEEE Region 10 Conference (31194) TENCON, 2013, pp.1-5.

10. L. Xia, L.G. Jiang, C. He, N. Nasser, S. Guizani, E. Al-Masri, A novel Fuzzy logic vertical handoff algorithm with aid of differential prediction and pre-decision method, 2007 IEEE International Conference on Communications, ICC'07, 2007, pp. 5665-5670.

11. S. Kabahuma, O.E. Falowo, Analysis of network operators revenue with a dynamic pricing model based on user behaviour in NGWN using JCAC, Proceedings of the Southern Africa Telecommunication Networks and Applications Conference (SATNAC), 2010, pp. 3258-3272.

12. Chandralekha, P.K. Behera, Optimization of vertical handoff performance parameters in heterogeneous wireless networks, Int. J. Modern Eng. Res. (IJMER) ISSN: 2249-6645, 1(2), (2011) pp. 597-601.

\section{AUTHORS PROFILE}

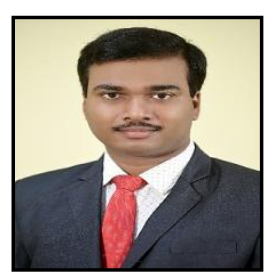

Sandeep Sivvam isworking as Assistant Professor in Dept. of E.C.E, Engineering\& Technology Program, Gayatri Vidya Parishad College for Degree and PG Courses (Autonomous), Rushikonda, Visakhapatnam, Andhra Pradesh, India. He received his B. Tech. in Electronics \& Communication Engineering and M. Tech. in VLSI Systems Design from JNTUK in 2010 and 2012, respectively. He has over 7 years of Teaching Experience. His area of research includes Wireless Communication and Embedded Systems.

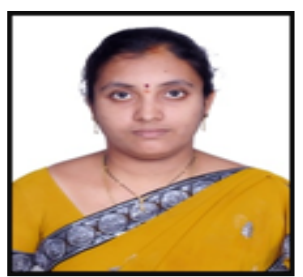

Dr. S. Neerajais working as Assistant Professor in the Department of Electronics and Communications Engineering, GITAM (Deemed to be University). She received $\mathrm{Ph}$. D degree from Andhra university in the year 2013. She has over 13 years of Teaching Experience. She has published more than 30 research papers in various reputed International/National journals/Conferences. Her area of Interest is Wireless and Mobile communications, Wireless Sensor Networks and CDMA/MIMO/OFDMA Wireless Communications. Currently, she is handling a DST-SERB research project.

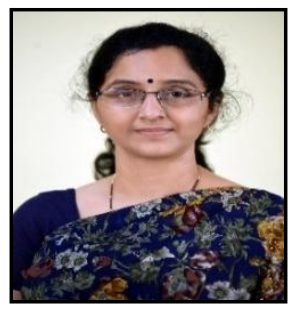

Jyothi Lakshmi T.S is working as Associate Professor in Dept. of E.C.E, Engineering \& Technology Program, Gayatri Vidya Parishad College for Degree and PG Courses (Autonomous), Rushikonda, Visakhapatnam, Andhra Pradesh, India. She received her B.Tech. in Electronics Engineering from REC, Calicut and M.E. in Electronics Engineering from Mumbai University in 1991 and 2004, respectively.She is also life Member of Institute of Electronics \& Telecommunication Engineers. She worked in reputed engineering colleges for the past 20 years and served in many positions. Her area of research includes Smart Antennas, Wireless Communication.

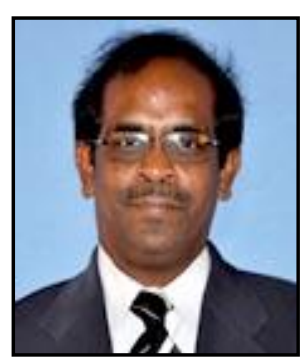

Prof. C. Dharma Raj is working as Professor in Dept. of E.C.E \& Principal (I/c), GITAM Institute of Technology (GIT), GITAM University, Visakhapatnam, Andhra Pradesh, India. He received his B.E. in Electronics \& Communication Engineering from Andhra University and M.E. in Microwave \& Radar Engineering from Osmania University in 1985 and 1987, respectively. He received his $\mathrm{Ph} . \mathrm{D}$ in 2012 from GITAM University. He worked as Scientist "B" in LRDE, Bangalore for about one year and later he worked in Marine and communication India Ltd., as a Technical officer. Eversince, after his work in industry he is working in GITAM University and served many positions for the past 33 years. He had published 26 papers in Reputed Journals like Springer, IEEE etc., and he also wrote 7 text books in the field of E.C.E and guided $10 \mathrm{Ph}$. D students and $28 \mathrm{M}$. Tech projects. His area of research includes Electromagnetic field theory, Antennas, Microwave \& Radar Engineering and Wireless Communications. 